\title{
CORRECTION
}

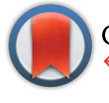

CrossMark

Cite this: J. Mater. Chem. A, 2017, 5, 1312

DOI: $10.1039 / \mathrm{cta}$ ta90258h

www.rsc.org/MaterialsA

\section{Correction: Local heterojunctions of atomic Pt clusters boost the oxygen reduction activity of RucoreaPdshell nanocrystallites}

\author{
Jeng Han Wang, ${ }^{a}$ Kuan-Wen Wang, ${ }^{\text {b }}$ Tian-Lin Chen, ${ }^{\mathrm{b}}$ Chia-Hsin Wang, ${ }^{\mathrm{c}}$ \\ Yu-Ming Huang, ${ }^{d}$ Yang-Yang Hsu ${ }^{d}$ and Tsan-Yao Chen*de \\ Correction for 'Local heterojunctions of atomic Pt clusters boost the oxygen reduction activity of \\ Rucore (Pdshell nanocrystallites' by Jeng Han Wang et al., J. Mater. Chem. A, 2016, 4, 17848-17856.
}

The original affiliations for the above manuscript are incorrect. Please see the correct affiliations below.

The Royal Society of Chemistry apologises for these errors and any consequent inconvenience to authors and readers. 